\title{
Comparison of Minimally Invasive Surgical Approaches in the Management of Small Renal Masses at a Safety Net Hospital
}

\author{
Liz Wang*, Michel Apoj, Remington Lim, Shaun Wason, Mark Katz, David Wang \\ Department of Urology, Boston Medical Center, Boston University School of Medicine, Boston, USA \\ Email address: \\ liz.wang@bmc.org (L. Wang), michel.apoj@bmc.org (M. Apoj), rlim1919@bu.edu (R. Lim), shaun.wason@bmc.org (S. Wason), \\ mark.katz@bmc.org (M. Katz), david.wang@bmc.org (D. Wang) \\ ${ }^{*}$ Corresponding author
}

\section{To cite this article:}

Liz Wang, Michel Apoj, Remington Lim, Shaun Wason, Mark Katz, David Wang. Comparison of Minimally Invasive Surgical Approaches in the Management of Small Renal Masses at a Safety Net Hospital. International Journal of Clinical Urology.

Vol. 5, No. 1, 2021, pp. 38-42. doi: 10.11648/j.jicu.20210501.18

Received: April 21, 2021; Accepted: May 3, 2021; Published: May 14, 2021

\begin{abstract}
Partial nephrectomy can be performed with traditional straight laparoscopy (SL), hand- assisted laparoscopic (HAL) and robotic-assisted laparoscopic (RAL) techniques. The purpose of this study is to analyze trends in surgical technique and compare the intra- and post-operative outcomes at a safety net hospital. We performed a single-institution retrospective review of intra- and post-operative outcomes in partial nephrectomy cases between 2012 and 2018. We analyzed the impact of patient demographics, tumor characteristics, and RENAL nephrometry score on surgical outcomes and post-operative complications among the three surgical approaches to partial nephrectomy. Of the 164 partial nephrectomies, 36 were SL, 65 HAL, and 63 RAL. Most SL was performed in the early years, whereas most RAL was performed in the later years, and HA was evenly distributed throughout the years. There was no difference in demographics, intra-operative complications, estimated blood loss, or positive margin rates between SL, HAL, and RAL. HAL partial nephrectomies had a higher RENAL nephrometry score and had statistically significant less warm ischemia and operative times when compared to RAL. HAL is a worthwhile technique in larger and more complex masses and especially in settings where robotic surgery is unable to be performed.
\end{abstract}

Keywords: Partial Nephrectomy, Laparoscopy, Robotics, Minimally Invasive Surgery, Renal Cell Carcinoma

\section{Introduction}

The evolution of surgical management for small renal masses is notable, as much has changed since the advent of radical nephrectomy $(\mathrm{RN})$ as the standard of care. $\mathrm{RN}$ is associated with higher rates of AKI and ESRD as well as greater reductions in eGFR when compared to partial nephrectomy (PN) [1]. RN also has a higher mortality rate when compared to PN in the setting of comparable cancerspecific survival between the two [2-4]. This shift away from RN can be attributed to its inherent inability to be nephronsparing.

Partial nephrectomy has largely become the standard of surgical care for the treatment of small renal masses. Minimally invasive PN options, including straight laparoscopy (SL), hand-assisted laparoscopy (HAL), and robot-assisted laparoscopy (RAL), have increasingly become utilized in different institutional settings [5, 6]. Laparoscopicbased techniques have gradually overshadowed the open approach, as they offer much of the same operational benefits with a noticeable decrease in morbidity. Several studies have demonstrated comparable outcomes between SL and open $\mathrm{PN}$ in regards to intraoperative complications, subsequent renal function, cancer-specific survival, positive surgical margins, and blood loss in the setting of shorter operation and hospital-stay lengths [7, 8]. SL was associated with no evidence of renal dysfunction and a $100 \%$ cancer specific survival at a 5-year follow up amongst patients with a localized renal tumor [9].

There are racial/ethnic and socioeconomic-related 
disparities in a patient's ability to access to these minimally invasive techniques for the management of small renal masses [6]. To our knowledge, there are no studies evaluating the trends of minimally invasive techniques for the management of small renal masses at a large safety net hospital. The purpose of this study is to analyze the trends in minimally invasive surgical techniques for $\mathrm{PN}$ and to compare the intra- and post-operative outcomes of these surgical techniques within a safety net hospital setting.

\section{Materials and Methods}

We performed a single-institution retrospective review of intra- and post-operative outcomes in minimally invasive PN cases at our safety net hospital between 2012 and 2018. Surgical approach with straight laparoscopic (SL), handassisted laparoscopic (HAL), or robotic-assisted laparoscopic (RAL) was surgeon- dependent. Statistical analysis included a linear regression model adjusting for age, gender, other patient demographics, RENAL nephrometry score, and tumor characteristics on pathology to investigate differences in estimated blood loss (EBL), warm ischemia time (WI), total operative time (OT), and length of hospital stay (LOS). Chisquare analysis was used to investigate differences in patient demographics and the rate of post-operative complications up to 30 days. Analysis of variances (ANOVA) was used to analyze differences in patient age, RENAL nephrometry scores, and positive margin rate among the three different surgical approaches.

\section{Results}

A total of 164 patients underwent PN with minimal invasive techniques between 2012 and 2018 of which 36, 65, and 63 surgeries were performed using the SL, HAL, and RAL technique, respectively. The majority of SL surgeries were performed in the early years $(68.6 \%$ between 2012 and 2013), while the majority of RAL were performed in the later, more recent years (92.1\% between 2014 and 2018) [Figure 1]. HAL cases were evenly distributed throughout the years reviewed.

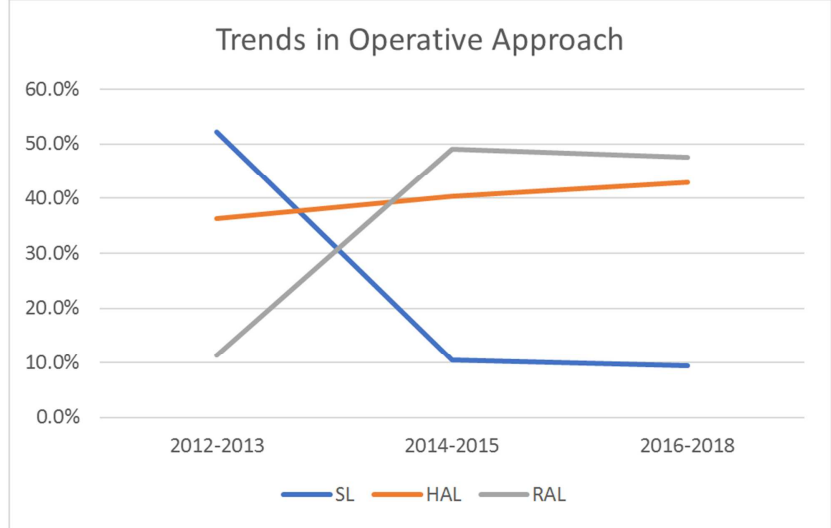

Figure 1. Trends in Minimally Invasive Operative Approach.

There were no statistically significant differences among the different surgical techniques in regards to age, gender, intra- operative complications, and positive margin rates ( $p>0.05$ ) [Table 1]. Partial nephrectomies performed with HAL had a statistically significant higher pre-operative RENAL Nephrometry score compared to RAL and SL $(\mathrm{p}<0.05)$ [Table 2]. One SL case was converted to open $(2.7 \%)$, and two RAL cases (3\%) had positive margins. There were no other intra-operative complications in any of the cases.

There was no difference in EBL between SL, HAL, and RAL (193, 291, and 259 min, respectively) ( $p>0.05)$. OT was longer for RA (229 min) compared to SL and HAL (187 and $198 \mathrm{~min}$, respectively) ( $\mathrm{p}<0.05)$. There was no statistical difference in OT between SL and HAL $(p>0.05)$. LOS was longer for HAL (3.1 days) when compared to SL and RAL (2.4 and 2.3 days, respectively) ( $<<0.05)$. There was no statistical significance difference in LOS between SL and RAL ( $\mathrm{p}>0.05)$. WI for HAL (19.6 min) was lower than for SL and RAL (21.3 and 25.3 min, respectively) $(\mathrm{p}<0.05)$. SL WI was lower than for RAL $(p<0.05)$ [Figure 2]. In regards to complications, HAL and SL had a higher overall incidence of Clavien-Dindo grade 3 and 4 post-operative complications when compared to RAL $(15.3 \%$ vs $13.8 \%$ vs $1.5 \%$, respectively) $(\mathrm{p}<0.05)$.

Table 1. Patient Characteristics: SL vs HAL vs RAL.

\begin{tabular}{|c|c|c|c|c|c|c|c|c|c|}
\hline & \multicolumn{2}{|l|}{ SL } & \multicolumn{2}{|c|}{ HAL } & \multicolumn{2}{|l|}{ RAL } & \multicolumn{2}{|c|}{ Total } & \multirow[t]{2}{*}{ p-value } \\
\hline & (N) & $\%$ & (N) & $\%$ & $(\mathbf{N})$ & $\%$ & $(\mathbf{N})$ & $\%$ & \\
\hline Total Patients & 36 & $22 \%$ & 65 & $40 \%$ & 63 & $38 \%$ & 164 & $100 \%$ & \\
\hline \multicolumn{10}{|l|}{ Age } \\
\hline$<50$ & 10 & $27.8 \%$ & 13 & $20.0 \%$ & 26 & $41.3 \%$ & 49 & $29.9 \%$ & \multirow{3}{*}{$>0.05$} \\
\hline $50-69$ & 23 & $63.9 \%$ & 41 & $63.1 \%$ & 28 & $44.4 \%$ & 92 & $56.1 \%$ & \\
\hline$\geq 70$ & 3 & $8.3 \%$ & 11 & $16.9 \%$ & 9 & $14.3 \%$ & 23 & $14.0 \%$ & \\
\hline \multicolumn{10}{|l|}{ Gender } \\
\hline Male & 18 & $50.0 \%$ & 41 & $63.1 \%$ & 35 & $55.6 \%$ & 94 & $57.3 \%$ & \multirow[t]{2}{*}{$>0.05$} \\
\hline Female & 18 & $50.0 \%$ & 24 & $36.9 \%$ & 28 & $44.4 \%$ & 70 & $42.7 \%$ & \\
\hline \multicolumn{10}{|l|}{ Demographics } \\
\hline Caucasian & 12 & $33.3 \%$ & 34 & $52.3 \%$ & 32 & $50.8 \%$ & 78 & $47.6 \%$ & \multirow{6}{*}{$>0.05$} \\
\hline Hispanic & 5 & $13.9 \%$ & 14 & $21.5 \%$ & 14 & $22.2 \%$ & 33 & $20.1 \%$ & \\
\hline African American & 15 & $41.7 \%$ & 12 & $18.5 \%$ & 13 & $20.6 \%$ & 40 & $24.4 \%$ & \\
\hline Native American & 1 & $2.8 \%$ & 1 & $1.5 \%$ & 1 & $1.6 \%$ & 3 & $1.8 \%$ & \\
\hline Cape Verde & 0 & $0.0 \%$ & 0 & $0.0 \%$ & 2 & $3.2 \%$ & 2 & $1.2 \%$ & \\
\hline Other & 3 & $8.3 \%$ & 4 & $6.2 \%$ & 1 & $1.6 \%$ & 8 & $4.9 \%$ & \\
\hline
\end{tabular}


Table 2. Tumor Characteristics: SL vs HAL vs RAL.

\begin{tabular}{|c|c|c|c|c|c|c|c|c|c|}
\hline & \multicolumn{2}{|l|}{ SL } & \multicolumn{2}{|c|}{ HAL } & \multicolumn{2}{|c|}{ RAL } & \multicolumn{2}{|c|}{ Total } & \multirow[t]{2}{*}{ p-value } \\
\hline & (N) & $\%$ & (N) & $\%$ & (N) & $\%$ & (N) & $\%$ & \\
\hline \multicolumn{10}{|c|}{ Size of Tumor (imaging) } \\
\hline$<5$ & 34 & $94.4 \%$ & 38 & $58.5 \%$ & 60 & $92.5 \%$ & 132 & $80.5 \%$ & \multirow[t]{2}{*}{$<0.05$} \\
\hline$\geq 5$ & 2 & $5.6 \%$ & 27 & $41.5 \%$ & 3 & $4.8 \%$ & 32 & $19.5 \%$ & \\
\hline \multicolumn{10}{|c|}{ Size of Tumor (pathology) } \\
\hline$<5$ & 34 & $94.4 \%$ & 40 & $61.5 \%$ & 57 & $87.7 \%$ & 131 & $79.9 \%$ & \multirow[t]{2}{*}{$<0.05$} \\
\hline$\geq 5$ & 2 & $5.6 \%$ & 25 & $38.5 \%$ & 6 & $9.2 \%$ & 33 & $20.1 \%$ & \\
\hline \multicolumn{10}{|c|}{ RENAL Nephrometry } \\
\hline Low & 31 & $86.1 \%$ & 41 & $63.1 \%$ & 50 & $79.4 \%$ & 122 & $74.4 \%$ & \multirow{3}{*}{$<0.05$} \\
\hline Intermediate & 5 & $13.9 \%$ & 23 & $35.4 \%$ & 13 & $20.6 \%$ & 41 & $25.0 \%$ & \\
\hline High & 0 & $0.0 \%$ & 1 & $2.0 \%$ & 0 & $0.0 \%$ & 1 & $0.6 \%$ & \\
\hline \multicolumn{10}{|l|}{ Tumor Type } \\
\hline $\mathrm{RCC}$ & 36 & $100.0 \%$ & 64 & $98.5 \%$ & 60 & $92.2 \%$ & 160 & $97.6 \%$ & \multirow[t]{2}{*}{$>0.05$} \\
\hline Benign & 0 & $0.0 \%$ & 1 & $1.5 \%$ & 3 & $4.8 \%$ & 4 & $2.4 \%$ & \\
\hline
\end{tabular}

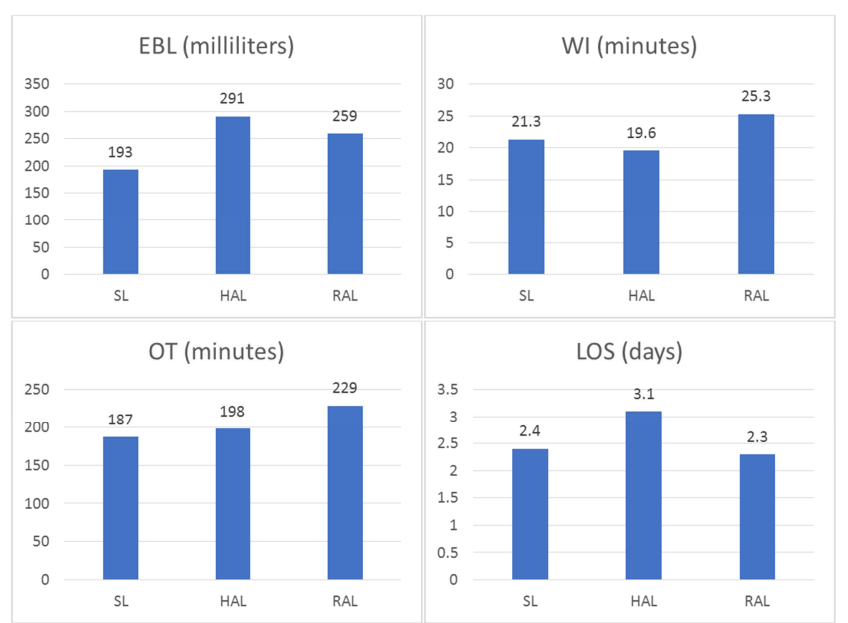

Figure 2. Estimated blood loss (EBL), operative time (OT), length of stay (LOS), and warm ischemia time (WI): SL vs HAL vs RAL.

\section{Discussion}

Kidney cancer is amongst the top ten most common malignancies affecting both men and women, with 14,830 deaths projected in the year 2020 [10]. Our increasing dependence on various imaging modalities has spurred an uptick of incidentally discovered small renal masses. Although there is a lack of recent data, it is projected that only $20 \%$ of small renal masses have benign pathology [11]. However, $65 \%$ of renal cancers present with exclusively local disease, and recent management and treatment have resulted in a favorable 5-year survival rate of 92.6\% [12].

Minimally invasive advancements within laparoscopy are emerging as the forefront of management of small renal masses. Several studies have demonstrated comparable outcomes between SL and open $\mathrm{PN}$ in regards to intraoperative complications, subsequent renal function, cancer-specific survival, positive surgical margins, and blood loss in the setting of shorter operation and hospital-stay lengths $[7,8]$. SL was associated with no evidence of renal dysfunction and a $100 \%$ cancer specific survival at a 5-year follow up amongst patients with a localized renal tumor [9]. HAL also exhibits reduced operation time, hospital stay, warm ischemia time, positive margins, and blood loss compared to open PN $[13,14]$.

In the current literature, comparisons between HAL and SL in the setting of PN seems to be uncharted territory. Studies between HAL and SL for radical nephrectomies indicate similar operative and post-operative parameters, including length of stay and post-procedural pain levels, while SL entails longer operative times [15-17]. RAL also holds great promise, as more facilities are incorporating this method [18, 19]. In 2010, the relative annual increase in the robotic PN approach was $45.4 \%$ [20]. RAL is associated with similar operation times, positive margins, and post- operative complications in comparison to SL [21, 22]. As a triad of negative margins, perioperative complications, and warm ischemia time $<25$ minutes, RAL scored higher than SL with better outcomes [23]. Although RAL and HAL are similar in regards to blood loss, positive margins, and complication rates, RAL necessitates higher costs [24].

In concordance with previous studies, our study did not demonstrate a statistically significant difference in intraoperative complications, EBL, or positive margin rate amongst the different minimally invasive surgical techniques used. OT was significantly longer for RAL and may be in part due to the significant amount of time dedicated to setting up the DaVinci robot that SL and HAL avoids. The $>30$ minute difference between RAL when compared to SL and HA should be taken into consideration when performing minimally invasive surgery in a medically high risk patient, when minimizing time under anesthesia is imperative to minimize operative complications.

It is worth noting that patients who underwent HAL had a longer hospital stay of about 1 day when compared to SL and RAL, which did not differ in LOS. However, it is notable that patients who underwent PN with HAL had a statistically higher pre-operative RENAL Nephrometry score, indicating these patients had inherently more anatomically complicated renal masses, thus potentially necessitating a more difficult surgical dissection, leading to increased rate of post-operative ileus and/or pain. However, this study was not designed to evaluate this difference. This increase in tumor complexity explains the increased rate of post- operative complications seen with HAL. Interestingly, HAL offered a statistically 
lower amount of warm ischemic time especially when compared with RAL (19.6 and 25.3 minutes, respectively), thus may be better suited for patients who need greater preservation of their renal function. The use of the additional hand port may assist in improved tissue handling when excising the renal mass, accounting for this difference in time. We suspect that the discrepancy between the groups may decrease over time as surgeons gain more confidence and experience with RAL for PN.

Our study shows that within our safety net hospital, the trend of minimally invasive technique for small renal masses shifted from mostly SL technique to RAL technique between 2012 and 2017, with nearly half of robotic PN for small renal masses performed in 2017 alone. Interestingly, HAL cases were evenly distributed across the years. This is likely attributed to surgeon-preference, as most HAL cases were performed by one particular surgeon at our center who has preference of HAL over SL technique.

Amongst our diverse patient population, we did not observe a statistically significant difference in age, gender, or demographics, including race and ethnicity, in regards to a particular surgical technique. Future studies comparing open vs. minimally invasive techniques for management of small renal masses at our safety net hospital paying special attention to race and ethnicity may further elucidate if there truly is a disparity in certain patients receiving minimally invasive techniques. However, our urology practice does not take insurance factors into account when determining surgical approach for management of renal masses, thus this disparity would not be expected.

The study is limited as it is retrospective in nature. More prospective studies comparing the three surgical techniques and additionally in comparison to open PN are needed to validate our results.

\section{Conclusions}

We have demonstrated that within our safety net hospital, HAL partial nephrectomies were done in more complicated cases and had statistically significant less warm ischemia and operative times when compared to RAL. The ability to perform HAL in these anatomically complex cases avoided the morbidity of having a flank incision in some cases where an open technique may have been considered. HAL may especially be a worthwhile technique in other safety net hospital settings and globally where the availability of performing robotic surgery is curtailed due to high costs and unavailability of performing robotic surgery.

\section{References}

[1] Sun M, Bianchi M, Hansen J, et al. Chronic Kidney Disease After Nephrectomy in Patients with Small Renal Masses: A Retrospective Observational Analysis. Eur Urol. 2012; 62: 696-703.

[2] Huang WC, Elkin EB, Levey AS, Jang TL, Russo P. Partial Nephrectomy Versus Radical Nephrectomy in Patients With
Small Renal Tumors-Is There a Difference in Mortality and Cardiovascular Outcomes? J Urol. 2009; 181: 55-62.

[3] Poppel H, Pozzo L, Albrecht W, et al. A Prospective, Randomised EORTC Intergroup Phase 3 Study Comparing the Oncologic Outcome of Elective Nephron-Sparing Surgery and Radical Nephrectomy for Low-Stage Renal Cell Carcinoma. Eur Urol. 2011; 59: 543-552.

[4] Pierorazio P, Johnson M, Patel H, et al. Management of Renal Masses and Localized Renal Cancer: Systematic Review and Meta-Analysis. J Urol. 2016; 162: 989-999.

[5] Permpongkosol S, Bagga HS, Romero FR, et al. Trends in the operative management of renal tumours over a 14 -year period. BJU Int. 2006; 98: 751-755.

[6] Xia L, Talwar R, Taylor BL, et al. National trends and disparities of minimally invasive surgery for localized renal cancer, 2010 to 2015. Urology. 2019; 37: 182. e17-182. e27.

[7] Marszalek M, Meixl H, Polajnar M, Rauchenwald M, Jeschke K, Madersbacher S. Laparoscopic and Open Partial Nephrectomy: A Matched-Pair Comparison of 200 Patients. Eur Urol. 2009; 55: 1171-1178.

[8] Gill IS, Kavoussi LR, Lane BR, et al. Comparison of 1,800 Laparoscopic and Open Partial Nephrectomies for Single Renal Tumors. J Urol. 2001; 178: 41-46.

[9] Lane BR, Gill IS. 5-Year Outcomes of Laparoscopic Partial Nephrectomy. J Urol. 2007; 177: 70-74.

[10] American Cancer Society Medical and Editorial Content Team. (2020). Key Statistics About Kidney Cancer. American Cancer Society. [Accessed 2020 December 20]. Retrieved from https://www.cancer.org/cancer/kidney-cancer/about/keystatistics.html\#written_by.

[11] Almassi N, Gill BC, Rini B, Fareed K. Management of the small renal mass. Transl Androl Urol. 2017; 6: 923-930.

[12] SEER. (2020). Cancer Stat Facts: Kidney and Renal Pelvis Cancer. National Cancer Institute, NIH. [Accessed 2020 $\begin{array}{llll}\text { December } & 20] \text {. Retrieved }\end{array}$ https://seer.cancer.gov/statfacts/html/kidrp.html.

[13] DeVoe WB, Kercher KW, Hope WW. et al. Hand-assisted laparoscopic partial nephrectomy after 60 cases: comparison with open partial nephrectomy. Surg Endosc. 2009; 23: 10751080 .

[14] Han K, Song G, You D, et al. Comparison of Hand-Assisted Laparoscopic vs Robot- Assisted Laparoscopic vs Open Partial Nephrectomy in Patients with T1 Renal Masses. J Endourol. 2017; 31: 374-379.

[15] Wolf JS, Moon TD, Nakada SY. Hand Assisted Laparoscopic Nephrectomy: Comparison to Standard Laparoscopic Nephrectomy. J Urol. 1998; 160: 22-27.

[16] Nelson CP, Wolf JS. Comparison of Hand Assisted Versus Standard Laparoscopic Radical Nephrectomy for Suspected Renal Cell Carcinoma. J Urol. 2002; 167: 1989-1994.

[17] Venkatesh R, Belani JS, Chen C, et al. Prospective Randomized Comparison of Laparoscopic and Hand-Assisted Laparoscopic Radical Nephrectomy. Urology. 2007; 70: 873877.

[18] Jabaji RB, Fischer H, Kern T. Trend of Surgical Treatment of Localized Renal Cell Carcinoma. Perm J. 2019; 23: 18-108. 
[19] Shah PH, Alom MA, Leibovich BC, et al. The Temporal Association of Robotic Surgical Diffusion with Overtreatment of the Small Renal Mass. J Urol. 2018; 200: 981-988.

[20] Ghani KR, Sukumar S, Sammon JD, Rogers CG, Trinh Q, Menon M. Practice Patterns and Outcomes of Open and Minimally Invasive Partial Nephrectomy Since the Introduction of Robotic Partial Nephrectomy: Results from the Nationwide Inpatient Sample. J Urol. 2014; 191: 907-913.

[21] Benway BM, Bhayani SB, Rogers CG, et al. Robot Assisted Partial Nephrectomy Versus Laparoscopic Partial Nephrectomy for Renal Tumors: A Multi-Institutional Analysis of Perioperative Outcomes. J Urol. 2009; 182: 866873.
[22] Aboumarzouk OM, Stein RJ, Eyraud R, et al. Robotic Versus Laparoscopic Partial Nephrectomy: A Systematic Review and Meta-Analysis Eur Urol. 2012; 62: 1023-1033.

[23] Zargar H, Allaf ME, Bhayani S, et al. Trifecta and optimal perioperative outcomes of robotic and laparoscopic partial nephrectomy in surgical treatment of small renal masses: a multi-institutional study. BJU Int. 2014; 116: 407-414.

[24] Elsamra SE, Leone AR, Lasser MS, et al. Hand-Assisted Laparoscopic Versus Robot-Assisted Laparoscopic Partial Nephrectomy: Comparison of Short-Term Outcomes and Cost. J Endourol. 2013; 27: 182-188. 